Revista de Negócios_ISSN 1980.4431_vol. 18, n.3, p.58_75, 2013_DOI:10.7867/1980431.2013v18n3p58_75

\title{
A cultura organizacional e a centralidade nas redes sociais: um estudo exploratório em uma empresa de serviços
}

\section{The organizational culture and centrality in social networks: an exploratory study in a service company}

Silvio de Oliveira Murdocco

Universidade Municipal de São Caetano do Sul - Brasil

s.murdocco@uol.com.br

Eduardo de Camargo Oliva

Universidade Municipal de São Caetano do Sul - Brasil

eduardo.oliva@uscs.edu.br

Recebido em 10 de maio de 2011. Alterado em 2 de fevereiro de 2013. Aprovado em 6 de fevereiro de 2013

Editor Responsável: Edson Roberto Scharf, Dr.

Processo de avaliação por double blind review

\section{Resumo}

O presente artigo apresenta uma pesquisa de campo sobre cultura organizacional e redes sociais que foi realizada junto a uma empresa prestadora de serviços. A questão central de pesquisa é: Como os valores da cultura organizacional se interligam as redes sociais? O objetivo foi verificar se existe a influência da cultura da organização nas características dos grupos sociais, com ênfase na centralidade. A literatura nacional e internacional evidencia que trabalhar com a centralidade significa identificar a posição em que o ator se encontra em relação às trocas e à comunicação na rede; a centralidade em uma rede traz a ideia de poder, isto é, quanto mais central é o indivíduo, mais bem posicionado ele está em relação às trocas e a comunicação como destacam Hanneman e Riddle (2005). Em relação aos procedimentos métodológicos, trata-se de um estudo exploratório de natureza quantitativo; o levantamento feito através de dois questionários elaborados por Nelson (2006) e Nelson e Vasconcelos (2007). O primeiro relativo à cultura organizacional denominado Perfil de Valores Agregados (PVA), que identifica a presença e as prioridades atribuídas dentro de uma organização quanto aos temas: Trabalho "tarefa", Relações "sociais", Controle "poder" e Cognição "Pensamento". O segundo relativo às redes formadas cujo instrumento constituiu-se da apresentação de uma lista com todos os participantes da pesquisa e foi solicitado a cada membro que informasse sobre a natureza dos contatos adjacentes a cada nome. Os campos nome e cargo já foram entregues pré-preenchidos pelos pesquisadores, os campos aprovações e número de e-mails recebidos e enviados foram preenchidos por meio de estimativa do respondente e o campo dependência foi respondido respeitando-se uma escala de 1 a 6 , onde 1 representava pouca e 6 muita dependência da outra pessoa. Utilizou-se uma amostra de 58 respondentes, todos funcionários próprios da empresa prestadora de serviços. Os 

serviços

dados foram tratados pela técnica estatística nãoparamétrica Mann Whitney para os valores da cultura e para os dados das redes Sociais os índices foram apurados por meio do software Ucinet $6.0 \mathrm{de}$ Borgatti, Everett, e Freeman (2002). Os resultados mostraram que os elementos centrais, ou seja, aqueles que têm o maior número de contatos na rede têm um maior alinhamento dos seus valores com os padrões da cultura organizacional e se concluiu que estes podem influenciar e participar na disseminação dos valores presentes na cultura organizacional. $\mathrm{O}$ que se pode perceber ao se analisar relação de nomes e funções dos colaboradores é que os quatro atores mais centrais da rede (centralidade) exercem funções gerenciais ou exercem algum tipo de liderança dentro da organização pesquisada; o primeiro é gerente de departamento pessoal, o segundo elemento mais central é gerente do departamento jurídico, o terceiro elemento mais central e sub-gerente operacional e trabalha diretamente com o Gerente Operacional, o quarto elemento é supervisor operacional. Os benefícios e a minimização de custos nas interações sociais caso a empresa utilize estes elementos centrais em programas de coaching ou mentoring, ou em outras ações de mudança, pode favorecer a retirada dos colaboradores da sua zona de conforto e ajudar a disseminar mais rapidamente a Missão, Visão e Valores Organizacionais.

Palavras-chave: cultura organizacional, redes sociais, centralidade e valores organizacionais.

\begin{abstract}
This article presents a field research about organizational culture and social network that was carried out in Service Company. The central question of this research is: How the values of organizational culture are interconnected with social networks? The goal was verify if there is an influence of the culture of the organization in the characteristic of the Social Groups, with emphasis in centrality. The national and international literature focuses that that to work with centrality means to identify the position of the actors are in relation to the exchanges and network communication; The centrality in the network brings the idea of power, that means, as much centered the person is, it has a better position in relation to exchanges and communication as highlight Hanneman e Riddle (2005). Regarding the methodological procedures this is a exploratory study with quantitative nature. The research was made with two set of questions that were elaborated by Nelson (2006) and Nelson e Vasconcelos (2007). The first is related to the organizational culture and is called Aggregated Values Profile (AVP), that identify the presence and priorities attributed inside the organization in relation to the
\end{abstract}

subjects: Work "task"; "social" Relations; "power" Control and Cognitions "thoughts". The second related to the formed network, whose tools are made by the presentation of a list with all the participants of the research and was asked to each member, information about the nature of nearest contact and each name. The places for name and position came already filled by the researchers, the place for approval and for the number of E-mails received and sent were filled by chance from the respondent, and the field dependency was answered considering the scale 1 to 6 , where 1 represents "fill" and 6 represents "a lot" dependency from another person. It was taken a sample of 58 respondents, all of them workers from the Service Company were the research was carried out. The data were worked out by the a non-parametric statistical technique, from Mann Whitney, to the cultural values, and the data for Social Network the numbers were found trough Ucinet 6.0 Software, from Borgatti, Everett and Freeman (2002). The results shows that the central points, that means, those that have a higher number of contact in the network, are more aligned with the values of the organizational culture. That means that they can influence and participate in the dissemination of values that are found in the organizational culture. What one can perceive when analyzing the list of the names and position of the workers is that that the four more centralized actors in the network have management position or have some kind of leadership in the researched organization. The first is manager of the personnel department, the second element is more central manager of the legal department, the third element most central and suboperations manager and works closely with the Operations Manager, the fourth element is operational supervisor. The benefits and the minimization of the costs in the social interactions if the company make use of those central elements in Coaching Program e or Mentoring, or in other changing actions, can help in bringing the workers out of the area of comfort and help in disseminate quickly the Mission, Vision and Values or the Organization.

Keywords: organizational culture, social network, network centrality and organizational values.

\section{Introdução}

A pesquisa realizada para a elaboração deste artigo teve origem nas observações empíricas dos pesquisadores de que a valorização da gestão de pessoas pode ser uma forma das empresas construírem e manterem a vantagem competitiva; buscou-se então, modelos de gestão com prática na inovação e 
criatividade que não se baseavam apenas em cálculos financeiros, mas que também, contemplavam e valorizavam os fatores humanos tanto individualmente como em equipe.

Dadas essas questões, Albuquerque (2002) diz que a área de Recursos Humanos é percebida tradicionalmente, como uma área dedicada às atividades administrativas, relacionadas com a gestão de benefícios, folha de pagamento, e outras tarefas rotineiras, mas que a partir da década de 90, enfrentou o desafio de se modernizar e se tornar agente de mudanças organizacionais.

Segundo Schein (2001), o planejamento de mudanças organizacionais deve ser mediado pela cultura organizacional, ou seja, quanto mais importante a ação de mudança for para a estratégia, maior deve ser sua compatibilidade com a cultura da organização. Nelson (2006) acrescenta as redes sociais de comunicação como mais um agente de mudanças e importante recurso que respalda a gestão organizacional por estudar os relacionamentos que fomentam o compartilhamento da informação e do conhecimento, Baldissera (2006) complementa afirmando que a comunicação organizacional é utilizada não só para impulsionar as transformações desejadas, mas também para preservar a identidade gerada pela cultura organizacional.

Pesquisar as SNA's Social Network Analisys ou análise de redes sociais envolvendo seus fluxos e atores, permite compreender a busca e análise de padrões como: força dos relacionamentos conforme Granovetter (1973) e Nelson e Vasconcelos (2007), densidade e faccionalismo de acordo Krackhartdt e Hanson (1993) e centralidade de redes que aprimoram a identificação dos atores mais influentes na rede sociais de comunicação nas pesquisas de Hanneman e Riddle (2005).

Para Kane e Borgatti (2011) verificar a centralidade de um ator significa identificar a posição em que ele se encontra em relação às trocas e à comunicação na rede; a centralidade em uma rede traz a ideia de poder, isto é, quanto mais central é o indivíduo, mais bem posicionado ele está em relação às trocas e a comunicação o que aumenta seu poder na rede, os autores fazem uma relação entre proficiencia e centralidade e dão o nome de $\mathrm{CP}$ (centrality and proficiency) e afirmam que os grupos terão um desempenho melhor nos seus fluxos de trabalho caso seus membros mais altamente proficientes forem centrais no fluxo de comunicação.

As organizações pesquisam a centralidade para saber quais de seus membros catalisam e disseminam as informações, uma vez que estes membros podem ser capazes de difundir a informação e influenciar os resultados. (ZHU et al., 2010). Os autores afirmam ainda que muitas vezes não é desejável transferir membros com elevado grau centralidade de suas redes, pois pode-se perturbar o movimento do conhecimento.

Essas ideias podem ser aplicadas desde redes pequenas até redes muito grandes e complexas que permitiriam identificar os nós da rede e avaliar a importância da sua posição para o atingimento dos objetivos organizacionais. (GÓMEZ et al., 2013).

Dos pesquisadores encontrados foram escolhidos (NELSON, 2001; 2003; 2005; 2006; NELSON e VASCONCELOS, 2007; HANNEMAN e RIDDLE, 2005), que vêm pesquisando cultura organizacional, valores e centralidade nas redes sociais de comunicação.

O objetivo deste artigo é identificar e analisar segundo a opinião dos funcionários de uma organização de serviços quais são os valores da cultura organizacional e quem dentre eles desempenha o papel de disseminação dos padrões da cultura organizacional dentro da rede social.

O objeto de estudo escolhido foi uma empresa prestadora de serviços, brasileira de médio porte com características 
A cultura organizacional e a centralidade nas redes sociais: um estudo exploratório em uma empresa de serviços

muito específicas, tanto por sua história e estrutura de capital, quanto por seu tamanho e diversidade de negócios. A escolha do segmento de serviços para a realização da pesquisa deve-se a relação serviçospessoas-cultura e redes de comunicação, como mostra a figura 1 .

Figura 1: Relação Valores da cultura organizacional, comunicação, serviço ao cliente.

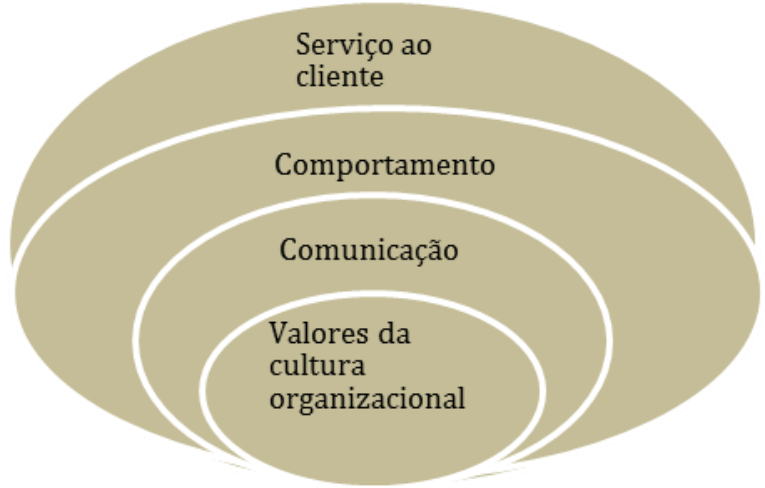

Fonte:Elaborado pelos pesquisadores com base em Nelson 2006

Nelson (2006), afirma que a cultura organizacional tende a influenciar na norma de comportamento ou no conjunto de valores que guiam a tomada de decisão do colaborador da empresa prestadora de serviços frente aos seus clientes. Desta forma, a participação das pessoas pode ser fundamental para que a empresa preste um serviço de qualidade ou não.

Como a cultura de uma empresa é sempre dinâmica e se renova cada vez que o seu sistema simbólico dá lugar a novos valores, crenças e significados, o conhecimento dos valores presentes na organização, o entendimento dos seus grupos sociais é relevante para que a empresa possa tomar ações preventivas e eficazes no sentido de diminuir a distância entre o que existe hoje e o que deve existir para que se tenha êxito, podendo assim ser uma das formas de as empresas construírem e manterem a vantagem competitiva.

A contribuição deste artigo para os estudos de SNA está na ampliação da aplicação dos estudos de Nelson (2006) e Nelson e Vasconcelos (2007), conectando valores da cultura organizacional com as redes sociais e a centralidade.
Este artigo foi estruturado da seguinte forma: a introdução que versa sobre as modificações que vêm acontecendo nos modelos de gestão organizacional. No referencial conceitual, destacam-se os conceitos sobre cultura organizacional, valores e o perfil dos valores agregados (PVA) de Nelson (2006) e as redes sociais. Nos procedimentos metodológicos destacam-se os procedimentos para a tabulação e análise dos dados quanto a quantificação do PVA e sua interpretação. Da mesma forma apresenta-se graficamente a rede social por meio do software Ucinet os elementos centrais e periféricos da rede. Por fim apresenta-se o cruzamento entre os resultados do PVA e redes sociais, finalizando com as conclusões e recomendações para futuras pesquisas.

\section{Referencial Conceitual}

Para organizar a apresentação do referencial teórico, optou-se pela criação da figura 2 que mostra a sequência dos conceitos abordados e as relações que serão utilizadas nas análises e conclusões.

Figura 2. Estrutura sequencial do referencial conceitual 


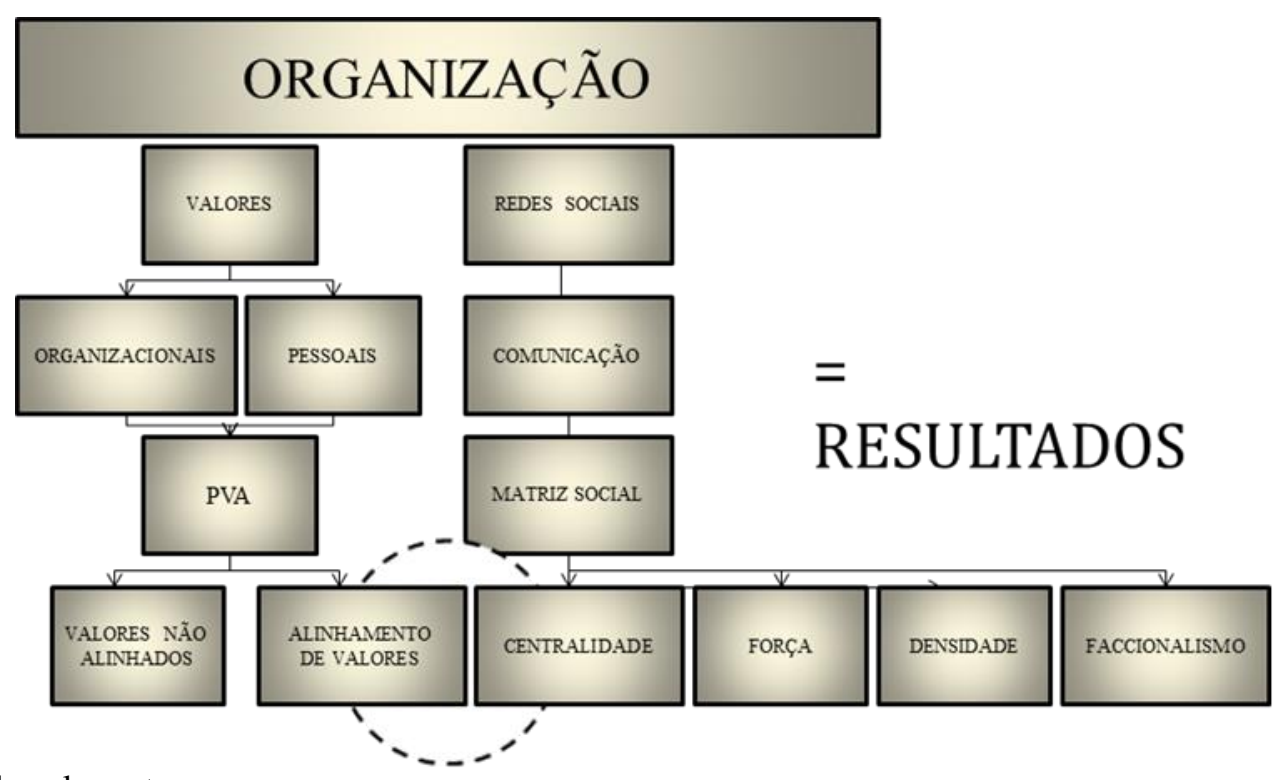

Fonte:elaborado pelos autores

Como mostra a figura 2, dentro de uma organização, os resultados são obtidos por aspectos múltiplos, a saber: tecnologia aplicada, decisões financeiras entre outras, porém conforme Nelson (2006) os valores da cultura organizacional e a capacidade de interação das pessoas nas redes sociais podem influenciar significativamente nos resultados. Nelson (2006) subdivide em valores pessoais e organizacionais. Para medir este fenômeno ele criou um instrumento denominado PVA - Perfil dos Valores Agregados que verifica $O$ alinhamento dos valores pessoais com os valores organizacionais sob a ótica do colaborador e não da organização.

Segundo Marineau (1992), a segunda vertente, redes sociais teve sua origem em Moreno que trabalhou com sociogramas e matrizes desde 1934. Para a identificação da matriz neste artigo se utilizou do modelo de Nelson e Vasconcelos (2007) formando a base para as análises e discussões. Dentre os elementos integrantes das redes sociais: faccionalismo, densidade, força e centralidade, optou-se por focar nos resultados da centralidade de redes, Hanneman e Riddle (2005) conectando-os ao perfil dos valores da cultura organizacional.

Portanto este artigo trata de redes sociais formais e amplia a aplicação do conceito para o entendimento dos elementos centrais da rede como disseminadores dos valores da cultura organizacional.

\subsection{A Cultura organizacional}

A cultura organizacional tem sido estudada e sugerida como objeto de estudo por inúmeros autores como Schein (1984, 1992 e 2001), Hofstede (1991,1994, 1995 e 2001), Thévenet (1985 e 2001), Nelson (2003) e no Brasil por Fleury (1991, 1996, 1997, 1999), Freitas (1991), Rodrigues (1997), Santos (1998), Barichello; Pozzobon, e Ribeiro (2003), Marchiori (2006) e Silva, Medeiros e Albuquerque (2008).

Freitas (1999) afirma que, as definições de cultura organizacional surgiram na década de 1960, com os conceitos de Desenvolvimento Organizacional (DO) que buscavam construir ou mudar organizações baseados na modificação das relações internas e implantação de valores compartilhados, no sentido moral e ético, o que estimularia o compromisso dos funcionários com a organização, substituindo a submissão e os controles burocráticos; já na década de 1980, Rodrigues (1997), salienta que 
A cultura organizacional e a centralidade nas redes sociais: um estudo exploratório em uma empresa de serviços

vários estudos foram realizados associando-se de um lado, à qualidade declinante da indústria americana e, de outro, o culto às empresas japonesas como exemplo marcante de organização e produtividade baseadas na sua cultura nacional.

Complementando, Barbosa (2002) salienta que, no período de 1980 a 1990 o conceito de cultura é reforçado devido à intensa competitividade entre as empresas norte americanas, européias e japonesas, sendo o sucesso dessas últimas atribuído à sua cultura, desta forma, a percepção da cultura aparece como sendo uma variável capaz de intervir na dinâmica dos negócios, dando-lhes valor e diferencial competitivo, sendo, portanto, vista como ativo estratégico.

Na concepção de Hofstede (1991), cultura é sempre fenômeno coletivo, que distingue os membros de um grupo (ou categorias de pessoas) uns dos outros, e pode ser aplicada a nações, organizações, profissões, grupos religiosos ou étnicos porque é, pelo menos, parcialmente, compartilhada com pessoas que vivem ou viveram no mesmo ambiente social onde ela foi assimilada, o autor divide os níveis da cultura organizacional em símbolos, heróis, rituais e valores e a visualização parte da camada mais superficial símbolos, para a camada mais profunda valores, formando o cerne da cultura.

Fleury (1996) argumenta que, para desvendar a cultura de uma organização, é preciso mapear as relações de poder nela presentes, sendo necessário considerar os processos de trabalho $\mathrm{e}$ as práticas administrativas, entre elas as de recursos humanos e de comunicação. Dessa forma, para conceituar cultura organizacional, a autora parte da concepção de Schein (2001), porém busca politizar a definição de cultura e fala que a cultura organizacional é concebida como um conjunto de valores e pressupostos básicos expressos em elementos simbólicos, que em sua capacidade de ordenar, atribuir significações, construir a identidade organizacional, tanto age como elemento de comunicação e consenso, como resulta e instrumentaliza as relações de dominação. (FLEURY; FISCHER, 1996).

Nelson (2006) define cultura organizacional como um mecanismo ou fronteira organizacional que determina $o$ que será valorizado pela empresa, ou seja, o autor classifica a cultura como uma fronteira organizacional, que ajuda a determinar quais as ideias e valores serão aceitos dentro da organização e quais serão excluídos.

\subsubsection{Valores}

Os valores são considerados por Schein (1992) e Hofstede (2001) como sendo o cerne da cultura organizacional e, portanto um dos elementos-chave para a sua analise; dentro da organização, existem os valores organizacionais e os valores pessoais, para Tamayo e Gondim (1996), os valores organizacionais podem ser definidos como princípios ou crenças, organizados hierarquicamente, relativos a tipos de estrutura ou a modelos de comportamento desejáveis que orientam a vida da empresa e estão a serviço de interesses individuais, coletivos ou mistos, já os valores pessoais para Freitas (2000) são formados a partir da convivência e das regras dos grupos sociais como família, escola, igreja e estão ligados às condições sociais, políticas e econômicas de cada um, são demonstradas externamente por meio de comportamentos e determinam este ou aquele modo de ser ou se um resultado é preferível do que o outro.

Para Nelson (2006), valores são convicções básicas que indicam a preferência por determinados estados e comportamentos, tais preferências possuem intensidades e interferem na disposição favorável ou desfavorável que temos em relação às coisas do mundo. Os valores são derivados da interação com os grupos sociais de referência do indivíduo, por tal razão, são internalizados com forte carga afetiva e passam a fazer parte do repertório da pessoa. A identificação dos valores individuais para o alinhamento aos valores 
organizacionais, portanto, pode prevenir eventuais conflitos decorrentes da ocorrência de discrepâncias entre os valores organizacionais (ou grupais) e valores dos indivíduos.

Hofstede (1991) defende que entre os elementos que formam a cultura, os valores são os mais afastados da observação direta, formando um núcleo de significados. Estes seriam transmitidos cedo na vida das pessoas, pelo convívio com a família, nas relações próximas de amizades de infância e na escola, fato que os tornam inconscientes, só podendo ser inferidos no comportamento observado.

Para Freitas (2000) a organização é uma extensão da personalidade dos seres humanos, sendo seus diferentes valores e significados provenientes das pessoas ou grupos que a constituem, pode-se dizer que parte da personalidade dos indivíduos se acha nelas representadas

\subsubsection{Perfil dos valores agregados}

Quando as pessoas entram para uma organização trazendo consigo valores pessoais e encontram os valores organizacionais que podem ser similares aos seus ou não, segundo Barret (2006) esta variável é chamada de "o alinhamento de valores e pode impactar diretamente nos resultados da empresa.
Nelson (2006) complementa e diz que as pessoas não podem lidar com mais de um pensamento ao mesmo tempo e que nossa capacidade de memória também é limitada, quando o ser humano recebe informações, ele escolhe, do imenso número de estímulos, um número relativamente pequeno de interesses e preocupações para atender, tendo por referência os valores pessoais.

Sendo assim, Nelson (2006) elaborou um instrumento que permite verificar se há ou não o Alinhamento entre os Valores Pessoais e os Valores Organizacionais definidos pela cultura da organização; a dificuldade na elaboração deste instrumento se deve ao dinamismo e variação da Cultura de organização para organização, desta forma, Nelson (2006) explica que se pode encontrar temas que estejam presentes em todas as organizações.

Foram estabelecidos por Nelson quatro temas, a saber: $1^{\circ}$.) Trabalho também conhecido como "tarefa" $2^{\circ}$.) Relações que derivam da comunicação; $3^{\circ}$.) Controle que é relativo a poder e a política e $4^{\circ}$.) Cognição ou Pensamento, que estão ligados à reflexão ou a racionalidade. $\mathrm{O}$ quadro 1 representa os temas e os subtemas do Perfil de Valor Agregado de Nelson(2006).

Quadro 1 - Temas e Subtemas do Perfil de valores agregados (PVA)

\begin{tabular}{|l|c|c|c|c|}
\hline Temas & Trabalho & Relações & Controle & Pensamento \\
\hline Subtemas & Esforço & Afeto & Dominância & Abstraçãão \\
\hline & Prazo & Empatia & Status & Planejamento/Organização \\
\hline & Terminar Tarefa & Sociabilidade & Politicagem & Exposição \\
\hline & Qualidade. & Lealdade. & Liderança & Flexibilidade \\
\hline
\end{tabular}

Fonte: Adaptado de Nelson (2006)

O quadro 1 mostra que cada tema é composto por quatro Subtemas, o tema Trabalho é composto pelos Subtemas: Esforço que trata do trabalho árduo ou do esforço constante; Prazo que lida com datas, horários e velocidade nas ações; Terminar Tarefa que trata da ênfase em concluir um projeto ou serviço apesar das outras pressões de tempo e Qualidade que lida com o valor percebido pelo cliente do produto do seu trabalho.

O tema Relações é composto pelo subtema Afeto que nos aproxima dos outros para podermos receber o calor humano, Empatia que nos coloca psicologicamente na situação do outro para poder dar o calor humano; a Sociabilidade que focaliza a interação e a atividade em 
A cultura organizacional e a centralidade nas redes sociais: um estudo exploratório em uma empresa de serviços

grupos ou equipes e a Lealdade que enfatiza as relações duráveis com obrigações recíprocas entre as pessoas ou coletivos.

No tema Controle encontram-se os subtemas, Dominância que envolve a imposição da vontade, sem muita justificativa ou pretexto; Status que favorece o indivíduo por criar ou frisar desigualdades simbólicas entre as pessoas; a Politicagem que envolve conseguir os objetivos pela manipulação e negociação, e a Liderança que atinge os objetivos indo à frente, inspirando ou persuadindo aos outros.

Finalmente o Tema do Pensamento composto pelos subtemas; Abstração que tende a lidar com conceitos intangíveis e generalidades; o Planejamento/Organização que se focalizam em detalhes; a Exposição que é ainda outra área que lida não com a produção das ideias, porém, com a sua transmissão e apresentação e a Flexibilidade que é uma dimensão pouco relacionada com as outras, mas é algo que surge consistentemente nas discussões sobre criatividade, adaptabilidade e capacidade de mudança.

Segundo Nelson (2006) ao aplicarse este instrumento de pesquisa pode-se perceber o nível de alinhamento entre os valores pessoais e organizacionais percebidos pelo colaborador comparando como o colaborador percebe os temas e subtemas na cultura da organização (valores organizacionais) e como ele gostaria que estes temas e subtemas fossem valorizados pela cultura organizacional (valores pessoais).

\subsection{Redes Sociais}

A comunicação para Marchiori (2006) é um dos instrumentos que possibilitam e dão continuidade ao processo de formação e manutenção da cultura de uma organização a partir da formação de grupos sociais, pois é através dos relacionamentos interpessoais que se disseminam os valores, as crenças, e atitudes; portanto, cultura e comunicação organizacional são aspectos indissociáveis em uma organização, ora a cultura influencia as redes sociais, ora as redes influenciam a cultura.

Para Nelson e Vasconcelos (2007) mapear as estruturas no sociograma permite ao pesquisador visualizar os canais de comunicação, identificar líderes e conexões entre as pessoas.

Abordar o tema redes sociais implica em integrar e relacionar conceitos que passam transversalmente por toda a área do comportamento organizacional, tais como, cultura, comunicação, estrutura, liderança ou mesmo motivação. Neste artigo o foco será apenas na sua vertente interna e formal, na qual se inserem as redes sociais da organização dos funcionários.

São várias as definições de Redes Sociais, porém diferentemente das abordagens tradicionais, Nelson e Vasconcelos (2007) comentam que a sociomatriz não só indica onde existem relacionamentos, como indica a intensidade ou frequência dos relacionamentos,

Para este artigo tomou-se como definição operacional o trabalho de Nelson e Vasconcelos (2007) enfatizando que as redes sociais são constituídas de uma série de elos que conectam agentes, neste caso, são grupos de pessoas que desenvolvem e mantêm contato para se comunicarem, normalmente acerca de algum interesse que partilham dentro das atividades diárias na organização.

Nelson (2006) afirma que quanto mais central é o ator na rede de comunicação de uma organização mais possibilidades ele tem de ocupar uma posição superior na hierarquia desta empresa, e mais alinhados devem ser seus valores pessoais aos valores organizacionais.

Hanneman e Riddle (2005) apresentam quatro denominações para centralidade: a primeira é a Centralidade de Informação (information centrality) que é a medida de centralidade que emprega a teoria de aproximação estatística (statistical estimation), baseada no concei- 
to de informação, usa uma combinação que analisa todos os caminhos entre os atores, para cada percurso analisado considera-se a informação contida no caminho correspondente.

A segunda é a Centralidade de Intermediação (betweenness centrality); que segundo Hanneman e Riddle (2005) considera um ator como meio para alcançar outros atores, visto que ele está posicionado nos caminhos geodésicos entre outros pares de atores na rede, ou seja, um indivíduo pode ter poucos contatos diretos na rede, estar conectado basicamente por ligações fracas, mas exercer um importante papel intermediando informações.

A terceira é a Centralidade de Proximidade (closeness centrality) cujo enfoque ressalta a distância de um ator em relação a outros, na rede, este enfoque está baseado na distância geodésica de cada ator com todos os demais, considerando-se as distâncias tanto as diretas quanto as indiretas, isto é, quanto mais próximo um ator estiver de outros atores da rede, mais central ele estará.

A quarta é a medida que foi utilizada neste artigo a Centralidade de Grau (degree centrality) que é o recurso que identifica o número de contatos diretos que um ator mantém em uma rede, é o que mede o nível de comunicação de um ator. Se um ator recebe muita informação ligações direcionadas a ele - diz-se que ele é proeminente ou tem prestígio na rede, ou seja, muitos outros atores buscam compartilhar informações com ele e isso pode indicar sua importância. Os atores que procuram outros - os que têm alto grau de saída de ligações - normalmente são atores influentes.

\section{Procedimentos Metodológicos}

Quanto a seu objetivo a pesquisa pode ser definida, segundo Gil (2002) como Exploratória, pois ela amplia os estudos de Nelson (2006, 2007), por meio do foco na centralidade e o PVA. Da mesma forma esta ampliação se verifica no trato de funções operacionais, diferentemente de Nelson que se dedicou ao nível gerencial.

No que diz respeito à fonte de dados pode-se defini-la como sendo de Campo, pois a coleta de dados foi realizada aonde o fato, fenômeno ou processo acontecem; é por natureza quantitativa pois pretende descobrir quantas pessoas da população a ser pesquisada compartilham de uma determinada característica

\subsection{Campo Empírico}

A pesquisa foi realizada em uma organização prestadora de serviços que franqueou $\mathrm{o}$ acesso dos pesquisadores, além de ter por características atuar há mais de vinte e cinco anos no segmento de terceirização de serviços, e contar com mais de sete mil empregados terceirizados em empresas localizadas na capital e grande São Paulo, além de ter uma administração corporativa composta por seis gerências bem definidas, estruturadas em espaços físicos distintos, com gerentes especializados em suas áreas de atuação a saber; Gerência Jurídica, Gerência de Recursos Humanos, Gerência do Departamento de Pessoal, Gerência Financeira, Gerência Operacional e Gerência Comercial que contavam com 140 colaboradores funcionários diretos do Grupo.

As gerências estudadas variam de tamanho e de localização física já que a organização está alocada em dois edifícios; durante a aplicação da pesquisa foram levantados os dados referentes ao número de colaboradores por gerência, para que se pudesse verificar de que forma estavam divididos os participantes.

O que se constatou é que a Gerência Operacional é composta por mais da metade dos colaboradores da área de administração corporativa do Grupo, fato que poderia invalidar ou pelo menos deixar tendenciosos os resultados dos testes estatísticos caso as seis gerências fossem comparadas, desta forma, o Grupo foi dividido da seguinte forma: Operacionais - 
A cultura organizacional e a centralidade nas redes sociais: um estudo exploratório em uma empresa de serviços

composto pelos colaboradores da Gerência Operacional - Não Operacionais - composto pelos colaboradores das outras cinco Gerências.

\subsection{Instrumentos de Coleta de Dados}

Foram utilizados dois instrumentos para a coleta de dados, um relativo à cultura organizacional denominado Perfil de Valores Agregados (PVA) de Nelson (2006) e outro Nelson e Vasconcelos (2007) relativo às redes sociais formadas pelos grupos de colaboradores seguindo-se o organograma da organização.

Os questionários são autoexplicativos e trazem orientações sobre o tempo e formas de preenchimento, os dados foram coletados utilizando-se o método ortodoxo de apresentar uma lista com todos os colaboradores da organização aos participantes da pesquisa e solicitar informações sobre a natureza dos contatos adjacentes a cada nome. O questionário é composto pelos campos Nome; Cargo; Aprovações; Dependência e Número de emails enviados. Os campos Nome e Cargo já foram entregues pré-preenchidos pelos pesquisadores, os campos Aprovações e Número de e-mails foram preenchidos por meio de estimativa do respondente e o campo Dependência foi respondido respeitando-se uma escala de 1 a 6 , onde 1 representa pouca e 6 muita dependência da outra pessoa.

\subsection{Procedimento para Tabulação e Análise dos Dados}

Para a tabulação foi utilizado o software Ucinet 6.0 de Borgatti, Everett, e Freeman (2002), que é um programa para análise de redes com o qual é possível montar uma matriz a partir dos códigos relatados pelos atores organizacionais nos formulários de pesquisa permitindo a análise estrutural das redes sociais, o seu tamanho, o papel dos atores dentro da rede, sua densidade, força, faccionalismo e a centralidade dos atores por meio de rotinas e funções de análise de vários atributos de redes pré-definidas e automatizadas baseadas em cálculos matemáticos.

Os dados coletados do PVA (Perfil de Valores Agregados) foram digitados em planilhas utilizando o software Excel for Windows da Microsoft (pacote Office) para depois serem exportados e analisados através do SPSS (Statistical Package for Social Science) e para o questionário de redes utilizou-se o Ucinet 6.0 de Borgatti, Everett, e Freeman (2002).

Para análise e tabulação dos dados obtidos com o questionário Perfil de Valores Agregados (PVA) de Nelson (2006) foi realizado um teste estatístico não-paramétrico, pois segundo Siegel e Castellan (2006) os testes estatísticos nãoparamétricos podem ser utilizados para analisar dados que estejam inerentemente classificados em ordem, bem como aqueles escores aparentemente numéricos, mas que tenham a força de ordem. Isto é, o pesquisador pode ser capaz de dizer, sobre os seus dados ou objetos, somente que um deles tem mais ou menos quantidade de certa característica do que outro, sem ser capaz de dizer quanto mais ou menos. No caso em questão, estudando o subtema lealdade, podemos ser capazes de estabelecer que o sujeito $A$ dá mais valor para o subtema lealdade que o sujeito $B$. Se os dados estão inerentes em ordem, ou mesmo, se eles podem ser categorizados somente com sinal de mais ou de menos (mais ou menos, melhor e pior), eles podem ser tratados por métodos nãoparamétricos.

Siegel e Castellan (2006), afirmam que um dos testes não-paramétricos é o teste de Mann Whitney, que pode ser usado para testar se dois grupos independentes foram extraídos de uma mesma população, este teste é uma alternativa muito útil para o teste paramétrico $t$ quando o pesquisador deseja evitar as suposições do teste $t$ ou quando a mensuração na pesquisa é mais fraca do que a dada em escala intervalar. Desta forma, para análise dos dados foi utilizado o teste estatístico não-paramétrico Mann Whitney. 
Primeiramente foi verificada a significância do teste para em seguida serem utilizadas as estatísticas (média mediana e desvio padrão ) encontradas nas respostas das assertivas dos temas $\mathrm{e}$ subtemas estudados; o Nível de Significância de um teste segundo Siegel e Castellan (2006) é a probabilidade de uma hipótese nula ser rejeitada, quando verdadeira, para este teste foi adotado como nível de significância 0.05 ( $\mathrm{p}<0.05)$.

Sendo assim, temos a Hipótese nula. HO: Não há diferença entre as distribuições da variável "i”" entre os grupos Operacional e Não-Operacional, onde o nível de significância é $\alpha=0,05$, portanto quando $\mathrm{p}<0,05$ rejeita-se a hipótese nula e pode-se dizer que há diferenças entre os grupos e quando $p$ $>0,05$ a hipótese nula não é rejeitada e não existe diferença significativa entre os grupos.
Cada tema, Trabalho - Relações Pensamento e Controle - recebeu uma pontuação que variou de (-48) a 48 de acordo com a somatória dos pontos atribuídos pelos respondentes em cada uma das assertivas que representam os temas, da mesma forma cada sub-tema variou de (-12) a 12 de acordo com a somatória dos pontos atribuídos nas assertivas que representam os sub-temas.

A pontuação foi obtida através da diferença entre a cultura organizacional ideal e a cultura organizacional percebidas pelo colaborador, pois, segundo Nelson (2006), são os valores pessoais que determinam a percepção da cultura. O quadro 2 mostra as pontuações possíveis em um sub-tema e em quais pontuações as expectativas do colaborador estão mais ou menos atendidas e ainda o zero onde existe o alinhamento dos valores pessoais e organizacionais.

Quadro 2: Diferença entre Cultura Ideal e Real percebida

\begin{tabular}{|c|c|c|}
\hline$(-12)(-11)(-10)(-9)(-8)(-7)(-6)(-5)(-4)(-3)(-2)(-1)$ & 0 & 123456789101112 \\
\hline Expectativas além das esperadas & $\begin{array}{c}\text { Alinhamento } \\
\text { dos valores }\end{array}$ & $\begin{array}{c}\text { Expectativas aquém das } \\
\text { esperadas }\end{array}$ \\
\hline
\end{tabular}

Fonte: elaborado pelos pesquisadores baseado em Nelson (2006).

No quadro 2 pode-se perceber que o resultado obtido através da diferença entre cultura organizacional Ideal e Real, quanto mais próximo de zero mais alinhados estarão os valores pessoais e os organizacionais, ou seja, as expectativas dos colaboradores estão atendidas e em sintonia com a ênfase que a cultura organizacional dá para o Tema ou o Subtema analisado.

Quando o resultado da diferença entre cultura organizacional Ideal e Real é um valor negativo, dentro dos limites de mais ou menos 48 para os Temas e mais ou menos 12 para os subtemas, significa segundo Nelson(2006), que as expectativas do colaborador sobre o Tema ou Subtema estão além das esperadas, ou seja, a cultura organizacional da mais ênfase para este
Tema ou Subtema do que o colaborador valoriza como ideal.

Mas quando o resultado da diferença entre cultura organizacional Ideal e Real é um valor positivo, significa segundo Nelson (2006), que as expectativas do colaborador sobre o Tema ou Subtema estão aquém das esperadas, ou seja, a cultura organizacional da menos ênfase para este Tema ou Subtema do que o colaborador valoriza como ideal.

Para que a relação expectativas, mais ou menos atendidas, fiquem mais claras, os números foram transformados em porcentagens, assim, um resultado de (12) passa a ser entendido como a expectativa $100 \%$ além da desejada e um resultado 12 passa a ser entendido como $100 \%$ aquém da desejada, quanto mais 
A cultura organizacional e a centralidade nas redes sociais: um estudo exploratório em uma empresa de serviços

próximo do zero mais alinhados os valores pessoais e organizacionais.

Nos questionários de Redes Sociais as respostas foram alocadas $\mathrm{N}$ por $\mathrm{N}$ por adjacência ou matrizes sociais, em que as linhas horizontais representam descrições do ego dos contatos com outros participantes da pesquisa e as colunas representam as descrições dos participantes da pesquisa sobre seus contatos com ego. Uma vez inseridas nas matrizes sociais, foi possível fazer diversas análises; no caso foi estudada a Centralidade das Redes.

Finalizando a análise foi realizado um cruzamento entre os resultados do PVA e o resultado encontrado quanto aos elementos mais centrais e mais periféricos da rede.

\section{Apresentação e Análise dos Resulta-}

Tabela 1: Número total de questionários tabulados

\begin{tabular}{l||c||c||c}
\multicolumn{1}{c|}{ Gerência } & $\begin{array}{c}\text { Número de } \\
\text { questionários } \\
\text { respondidos }\end{array}$ & $\begin{array}{c}\text { Número de } \\
\text { questionários } \\
\text { descartados }\end{array}$ & $\begin{array}{c}\text { Número de } \\
\text { questionários tabulados }\end{array}$ \\
\hline \hline Jurídico & 5 & 2 & 3 \\
Rec. Humanos & 7 & 5 & 2 \\
Dep. Pessoal & 11 & 2 & 9 \\
Financeiro & 8 & 2 & 6 \\
Comercial & 7 & 2 & 33 \\
Operacional & 71 & 38 & $\mathbf{5 8}$ \\
Total & $\mathbf{1 0 9}$ & $\mathbf{5 1}$ & 5 \\
\hline
\end{tabular}

Fonte: dados de pesquisa.

Como mostra a tabela 1 houve um número significativo de questionários descartados, pois a pesquisa foi realizada com todos os colaboradores presentes, independentemente da posição hierárquica e grau de instrução, notou-se que para alguns dos participantes com pouca escolaridade os questionários foram preenchidos com erros dificultando sua utilização.

\subsection{Resultados dos questionários do}

$\operatorname{dos}$

A apresentação dos resultados da pesquisa está estruturada da seguinte forma: Caracterização da amostra, alinhamento dos valores pessoais $\mathrm{e}$ organizacionais PVA, resultado da centralidade da rede, mostrando os elementos centrais e por fim o cruzamento do resultado do alinhamento de valores percebidos pelos atores centrais da rede frente aos periféricos da rede estudada.

\subsection{Caracterização da amostra}

A tabela 1 mostra que do total de 140 colaboradores indicados pela gerência de RH, 109 estavam presentes e tiveram a oportunidade de responder aos dois questionários. 
Tabela 2: Comparação dos resultados dos Grupos Operacional e Não Operacional em relação aos temas:

\begin{tabular}{|c|c|c|c|c|c|}
\hline & \multicolumn{2}{|c|}{ Operacional } & \multicolumn{2}{|c|}{ Não Operacional } & \multirow[b]{2}{*}{ Significância do Teste ${ }_{1}$} \\
\hline & Média & $\begin{array}{l}\text { Desvio } \\
\text { Padrão }\end{array}$ & Média & $\begin{array}{l}\text { Desvio } \\
\text { Padrão }\end{array}$ & \\
\hline $\begin{array}{l}\text { Trabalho } \\
\text { (-48 a } 48 \text { pontos) }\end{array}$ & 3,6 & 6,3 & 10,1 & 7,5 & $\mathrm{p}<0,05$ \\
\hline $\begin{array}{l}\text { Relações } \\
\text { (-48 a } 48 \text { pontos) }\end{array}$ & 5.8 & 9.7 & 6.7 & 9.2 & $\mathrm{p}>0,05$ \\
\hline $\begin{array}{l}\text { Controle } \\
(-48 \text { a } 48 \text { pontos) }\end{array}$ & -5.1 & 11.4 & -12.0 & 12.3 & $\mathrm{p}<0,05$ \\
\hline $\begin{array}{l}\text { Pensamento } \\
\text { (-48 a } 48 \text { pontos) }\end{array}$ & -1.2 & 9.3 & 1.0 & 10.0 & $\mathrm{p}>0,05$ \\
\hline
\end{tabular}

Fonte: dados da pesquisa.

Como mostra a tabela 2, o tema Trabalho apresenta um nível de significância $\mathrm{p}<0,05 \mathrm{e}$, portanto se pode rejeitar a hipótese nula $\mathrm{H} 0$, o quer dizer que existe uma diferença significativa entre os dois grupos; a Média do grupo Operacionais para o tema Trabalho é 3,6, o que significa que neste tema as expectativas do grupo estão $7,5 \%$ aquém da desejada, ou seja, a cultura organizacional percebida está bem próxima da desejada pelos colaboradores, fato que para Nelson (2006) representa que a cultura organizacional enfatiza o Trabalho e o Grupo dos colaboradores Operacionais percebe esta ênfase, mas gostaria que ela fosse um pouco maior.

Já o grupo dos Não Operacionais, a Média para o tema Trabalho é 10,1 o que significa que neste tema as expectativas do grupo estão $21 \%$ aquém da desejada, é quase três vezes a Média do grupo dos Operacionais, ou seja, a cultura organizacional percebida está significativamente distanciada da desejada pelos colaboradores.

Esta diferença entre os dois grupos é bastante interessante, na medida em que o grupo dos Operacionais se sente mais atendido no tema Trabalho que o grupo dos Não Operacionais, fato que pode denotar uma maior preocupação da organização em proporcionar melhores condições de Trabalho para o grupo dos Operacionais pela característica das suas tarefas voltadas a operacionalização e deixa um pouco de lado este tema para os Não Operacionais, que se mostraram insatisfeitos, ou não atendidos neste tema.

Continuando a análise da tabela 2 podemos perceber que, assim como o Tema Trabalho, o Tema Controle apresenta um nível de significância $\mathrm{p}<0,05$ e, portanto se pode rejeitar a hipótese nula $\mathrm{H} 0$, o quer dizer que existe diferença significativa entre os dois grupos, outro fato a se destacar é que neste Tema aparecem resultados negativos.

Este fato pode representar segundo Nelson (2006) que os colaboradores percebem que existe uma maior ênfase da Cultura Organizacional no Controle do que os colaboradores gostariam, além de negativos os resultados apresentam valores significativamente altos, a Média do grupo dos Não Operacionais é a mais alta de todos os outros resultados (-12) o que significa que para este grupo a cultura organizacional da uma ênfase para o controle $25 \%$ além da desejada, e para o grupo dos Operacionais a Média é 5,1, o que significa que a cultura organizacional dá uma ênfase $10 \%$ além da desejada. Pode parecer normal $o$ fato de os colaboradores acharem que a Cultura da empresa dá mais ênfase ao controle do que eles gostariam, mas o que chama a atenção nestes dados é que o grupo dos Não Operacionais percebem muito mais $\mathrm{o}$ controle do que os Operacionais, diferença de $25 \%$ para $10 \%$. 
A cultura organizacional e a centralidade nas redes sociais: um estudo exploratório em uma empresa de serviços

\subsection{Análise dos Resultados Referentes a Redes Sociais}

A tabela 3 mostra os resultados obtidos para os dez atores mais centrais e os dez mais periféricos.

Tabela 3: Resultado da Centralidade Grupo

\begin{tabular}{c||c||c||c||c||c|cc}
\hline \multicolumn{4}{c||}{ Elementos Centrais da Rede } & \multicolumn{4}{c}{ Elementos Periféricos da Rede } \\
\hline \hline & Nome & $\begin{array}{c}\mathbf{N}^{\circ} \text { de } \\
\text { Contatos }\end{array}$ & $\begin{array}{c}\mathbf{\%} \text { de } \\
\text { Contatos }\end{array}$ & & Nome & $\begin{array}{c}\mathbf{N}^{\circ} \text { de } \\
\text { Contatos }\end{array}$ & $\begin{array}{c}\text { \% de } \\
\text { Contatos }\end{array}$ \\
\hline \hline $\mathbf{1}$ & H Z & 54.0 & 94.7 & $\mathbf{1}$ & R D S & 4.0 & 7.0 \\
\hline $\mathbf{2}$ & T C & 51.0 & 89.5 & $\mathbf{2}$ & J M & 8.0 & 14.0 \\
\hline $\mathbf{3}$ & A P & 50.0 & 87.7 & $\mathbf{3}$ & O C & 8.0 & 14.0 \\
\hline $\mathbf{4}$ & A C & 47.0 & 82. & $\mathbf{4}$ & A P S & 11.0 & 19.3 \\
\hline $\mathbf{5}$ & M G & 45.0 & 78.9 & $\mathbf{5}$ & A CR & 11.0 & 19.3 \\
\hline $\mathbf{6}$ & I D & 43.0 & 75.4 & $\mathbf{6}$ & F D M & 12.0 & 21.1 \\
\hline $\mathbf{7}$ & M D & 41.0 & 71.9 & $\mathbf{7}$ & N J & 12.0 & 21.1 \\
\hline $\mathbf{8}$ & D P & 41.0 & 71.9 & $\mathbf{8}$ & M D S & 13.0 & 22.8 \\
\hline $\mathbf{9}$ & J Q & 40.0 & 70.2 & $\mathbf{9}$ & P B & 16.0 & 28.1 \\
\hline $\mathbf{1 0}$ & J J & 39.0 & 68.4 & $\mathbf{1 0}$ & R P & 16.0 & 28.1 \\
\hline
\end{tabular}

Fonte:dados da pesquisa.

A tabela 3 mostra quem são os atores que tem o maior número de contatos diretos na rede, mostra também que o número máximo de contatos é 54 e o número mínimo de contatos é 4 , na coluna porcentagem dos contatos encontram-se os resultados de quanto percentualmente representa o número de contatos de cada um dos atores, a média de contatos é 28,1 .

O Software Ucinet 6.0 disponibiliza um outro recurso que desenha a rede possibilitando uma melhor visualização da centralidade chamado de NetDraw; a figura 1 mostra o desenho de rede do tipo centro-periferia onde aparecem os elementos centrais ou aqueles que têm o maior número de ligações ou contatos e os que se encontram na periferia e têm o menor número de ligações.

Figura 3. Desenho da rede do Grupo

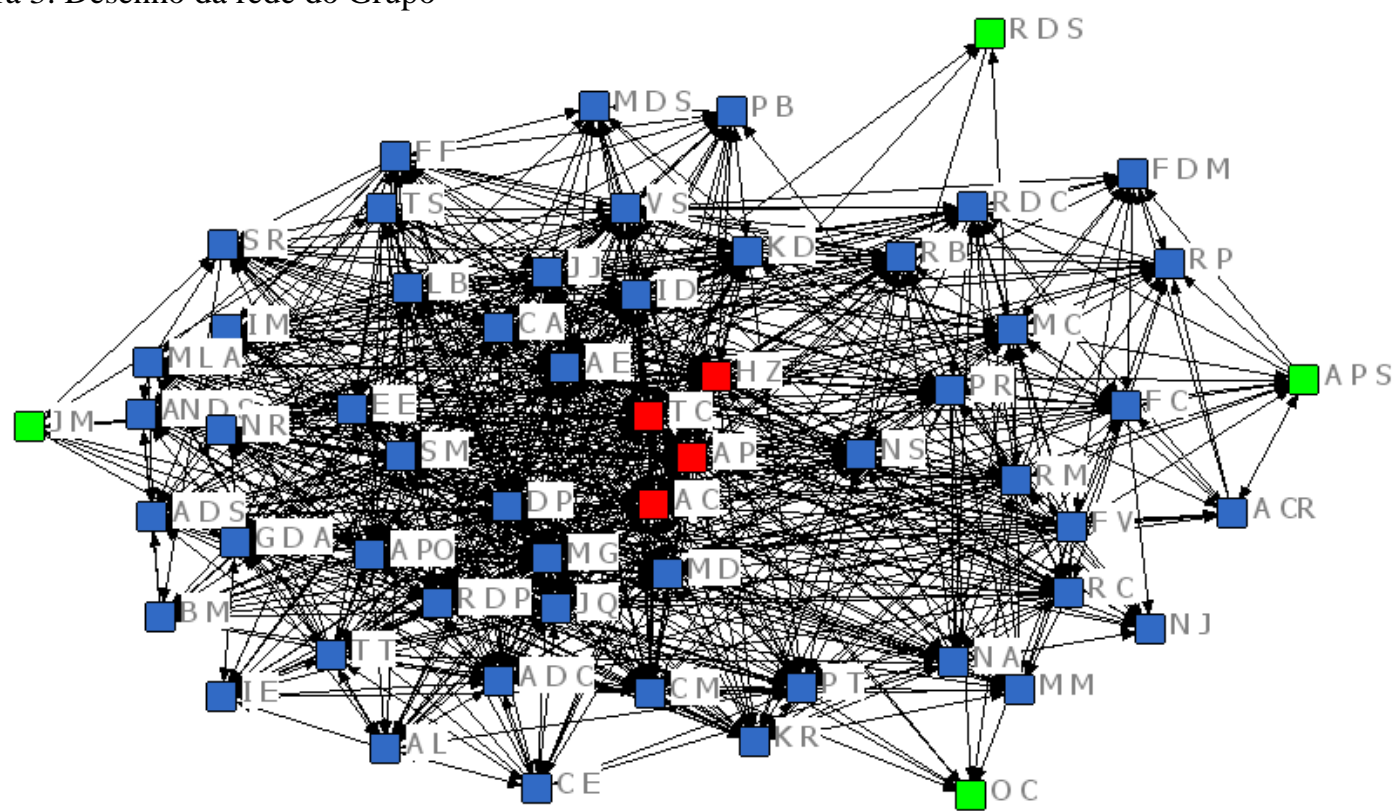

Fonte:dados da pesquisa.

No resultado da centralidade obtido, pode-se perceber que os elementos que mais têm ligações aparecem no centro da Rede, enquanto os que têm um número 
menor de contatos aparecem na periferia. Identificou-se que o alto número de contatos ficou circunscrito aos quatro atores que exercem as seguintes funções: dois deles são gerentes de departamento, um é Sub-Gerente operacional, e o outro é Supervisor.

A medida identificada neste artigo foi a Centralidade de Grau (degree centrality) que é o recurso que identifica o número de contatos diretos que um ator mantém em uma rede. Esta centralidade de redes segundo Hanneman e Riddle (2005) pode significar que estes elementos recebem muita informação - ligações direcionadas à eles - diz-se que eles são proeminentes ou tem prestígio na rede, a centralidade é geralmente identificada com situações de concentração de poder, decisão e controle dos recursos.

\subsection{Cruzamento do PVA e Redes Sociais}

Para responder ao alinhamento dos valores organizacionais com a centralidade, conforme apontado na figura 2 elaborou-se a tabela 4 que apresenta o resultado do PVA para os elementos mais centrais e os mais periféricos da rede da empresa pesquisada.

Tabela: 4 Resultados do PVA para centralidade das redes.

\begin{tabular}{|c|c|c|c|c|c|c|c|c|c|c|}
\hline Redes & \multicolumn{5}{|c|}{ Elementos Centrais } & \multicolumn{5}{|c|}{ Elementos Periféricos } \\
\hline PVA & $\mathrm{HZ}$ & PR & $\mathrm{AP}$ & MG & Média & RDS & $\mathrm{OC}$ & JM & NA & Média \\
\hline Trabalho & 3 & 2 & 6 & 1 & 3,0 & 8 & 12 & 11 & 10 & 10,75 \\
\hline Relações & 2 & 3 & 5 & 1 & 2,75 & 9 & 12 & 8 & 10 & 9,75 \\
\hline Controle & -4 & -3 & -5 & -2 & $-3,50$ & -12 & -7 & -8 & -8 & -9 \\
\hline Pensamento & 1 & 1 & 1 & -1 & 0,75 & 6 & 5 & -2 & 9 & 4,5 \\
\hline
\end{tabular}

Fonte: dados da pesquisa

A tabela 4 mostra que a média dos atores centrais da rede é bem próxima de zero, o que significa que seus valores estão bem alinhados aos valores da Cultura Organizacional, já a média dos atores periféricos está significativamente afasta do zero ou os valores pessoais dos atores estão menos alinhados aos da Cultura Organizacional.

Os resultados obtidos por meio da aplicação do questionário de valores pessoais o PVA de Nelson (2006) mostraram a visão dos colaboradores mais centrais da rede sobre a Cultura Organizacional que está sendo disseminada no grupo, portanto, no que tange aos quatro temas Trabalho, Relações, Controle e Pensamento, a Cultura organizacional está bem próxima à desejada, ou seja, está tendendo a zero.

Para os elementos periféricos da rede todos os temas se apresentam significativamente afastado do zero o que mostra o não alinhamento ou uma percepção mais distante dos valores organizacionais.

Pode-se perceber também que os quatro atores mais centrais da rede exercem funções gerenciais ou exercem algum tipo de liderança dentro da Organização pesquisada como afirmam Hanneman e Riddle (2005), Nelson e Vasconcelos (2007), Zhu et al., (2010), Kane e Borgatti (2011) e Gómez et al., (2013), quando dizem que a centralidade pode identificar situações de concentração de poder, decisão e controle dos recursos.

Aquele que é o mais central e tem o maior número de contatos na rede é gerente de departamento pessoal, o segundo elemento mais central é gerente do departamento jurídico, o terceiro elemento mais central e sub-gerente operacional e trabalha diretamente com o Gerente Operacional, o quarto elemento é supervisor operacional, todos com a percepção da cultura bem alinhada; já os elementos periféricos exercem funções operacionais, dois são 
A cultura organizacional e a centralidade nas redes sociais: um estudo exploratório em uma empresa de serviços

motoristas uma é auxiliar de almoxarifado e a ultima é secretária.

\section{Conclusão}

Os valores pessoais dos elementos centrais da rede estão bem próximos aos valores percebidos na Cultura da Organização, fato evidenciado nos elementos mais centrais da rede, pois desempenham funções gerenciais e pressupõe-se tenham seus valores pessoais mais alinhados aos da cultura organizacional. Por este aspecto eles têm a tendência a disseminar esses valores nos contatos que mantém dentro da rede social.

Também percebe-se que na empresa pesquisada estes elementos mais centrais podem maximizar os benefícios das ações gerenciais, pela centralidade que possuem na rede formal dentro dos grupos de trabalho, minimizando os conflitos e os custos das interações disformes. Nelson e Vasconcelos (2007), sugerem que a empresa possa utilizar estes elementos centrais em programas de coaching ou mentoring ou ainda como elementos facilitadores em processos de mudanças organizacionais ou em ações estratégicas que venham a retirar os colaboradores da sua zona de conforto, mas principalmente como líderes dos grupos multifuncionais com o objetivo de disseminarem mais rapidamente a Missão, Visão e Valores Organizacionais em busca dos resultados desejados pela organização.

No que diz respeito a novas pesquisas sobre o tema, seria interessante que fosse ampliado o número de empresas pesquisadas no segmento de serviços, a fim de se verificar como se dá, por exemplo, a questão da centralidade das redes em organizações de diferentes portes e ramos de negócios para se verificar se os temas do PVA se repetem quanto ao ciclo de alinhamento dos valores e se os elementos mais centrais encontram-se entre aqueles das funções gerenciais centralidade na rede - que possuem maior possibilidade de disseminação dos valores da cultura organizacional.

Outra proposta seria pesquisar o tema em empresas de culturas nacionais diferentes, mas do mesmo segmento, e verificar se há ou não diferença da percepção dos valores entre culturas de diferentes países.

\section{Referências}

ALBUQUERQUE, L. G. A gestão estratégica de pessoas. In: FLEURY, Maria T. L. (Coord.). As pessoas na organização. São Paulo: Gente, 2002.

CROSS, R.; PARKER, A.; BORGATTI, S. P. A bird's-eye view: using social network analysis to improve knowledge creation and sharing. Knowledge Directions, v.2, n.1, p.48-61, 2000. Disponível em: http://www.analytictech. com/borgatti/publications.htm. Acesso em 13 abril. 2010.

BARBOSA, L. Cultura e empresas. Rio de Janeiro: Jorge Zahar, 2002.

BARICHELLO, E.; POZZOBON, C. M.; RIBEIRO, M. B. Comunicação informal e cultura organizacional. Comunicação Organizacional - FAMECOS-PUCRS, Porto Alegre, v. 2, 2003.

BARRET, R. Buildinga values-driven organization: A whole system approach to cultural transformation. Oxford: Elsevier, 2006.

BORGATTI, S. P.; EVERETT, M. G.; FREEMAN, L. C. UCINET for Windows: software for social network analysis. Boston: Harvard Analytic Technologies, 2002.

BORGATTI, S. P.; EVERETT, M. G. Network analysis of 2-mode data. Social Networks, v. 19, p. 243 -269, 1997.

BORGATTI S.P. Centrality and network flow. Social Networks. V. 27, n. 1, p. 5571, 2005. 
FISCHER II, R. M. Mudança e transformação organizacional. In: FLEURY, M. T. L. (Coord.) As pessoas na organização. São Paulo: Gente, 2002.

FLEURY, M. T. L. (Coord.) As pessoas na organização. São Paulo:Gente, 2002.

FLEURY, M. T. L. Cultura organizacional e estratégias de mudanças: recolocando estas questões no cenário brasileiro atual. Revista de Administração, São Paulo, v. 26, n. 2, p. 3-11, 1991.

FLEURY, M. T. L. O desvendar da cultura de uma organização - uma discussão metodológica. In: FLEURY, M. T. L.; FISCHER, Rosa M. (coords.). Cultura e poder nas organizações. 2 ed. São Paulo: Atlas, 1996.

FLEURY, M. T. L.; SHINYASHIKI, G. T.; STEVANATO, L. A. Arqueologia teórica e dilemas metodológicos dos estudos sobre cultura organizacional. In: MOTTA, F. C. P.; FREEMAN, L.C., Centrality in networks: Conceptual clarification. Social Networks 1, p. 215239, 1979.

FREITAS, M. E.; FLEURY M. T. L. Cultura organizacional: formação tipologias e impactos. São Paulo: MakronMcGraw-Hill, 1991.

FREITAS, M. E. Cultura organizacional: identidade, sedução e carisma? Rio de Janeiro: FGV, 1999.

FREITAS, M. E. Contexto social e imaginário organizacional moderno. Revista de Administração de Empresas, São Paulo, v. 40, n. 2, p. 6-15, abr.jun. 2000.

GIL, A. C. Como elaborar projeto de pesquisa. 4. ed. São Paulo: Atlas, 2002.

GÓMEZ D.; FIGUEIRA J. R.; EUSÉBIO A. Modeling centrality measures in social network analysis using bi-criteria network flow optimization problems. European Journal of Operational Research, n. 226, p.354-365, 2013.
HANNEMAN, R. A.; RIDDLE M. Introduction to social network methods. Riverside: University of California, 2005. Disponível em: <http://faculty.ucr. edu/ hanneman/>. Acesso em: 28 set. 2009.

HOFSTEDE, G. Culture's consequences: international differences in workrelated values. London: Sage Publications, 1980.

HOFSTEDE, G. Cultures and organizations: software of the mind. London: McGraw-Hill, 1991.

HOFSTEDE, G. The business of international business is culture. International Business Review, Great Britain, v. 3, n. 1, p. 1-14, 1994.

HOFSTEDE, G. The interaction between national and organizational value systems. Journal of Management Studies, v. 22, n. 4, p. 347-57, 1995.

HOFSTEDE, G. Culture's consequences: comparing values, behaviors, institutions, and organizations across nations. 2 ed. California: Sage Publications, 2001.

KANE, G. C.; BORGATTI, S. P. Centrality-IS Proficiency Alignment MIS Quarterly. v, 35, n. 4, p. 1063-1078, December, 2011.

MARCHIORI, M. Cultura e comunicação organizacional: uma perspectiva abrangente e inovadora na proposta de interrelacionamento organizacional. In: MARCHIORI, M. (Org.). Faces da cultura e da comunicação organizacional. São Caetano do Sul: Difusão, 2006.

MARINEAU, R. F. Jacob Levy Moreno 1889-1974: Pai do psicodrama, da sociometria e da psicoterapia de grupo. São Paulo: Ágora, 1992.

NELSON, R. E. Social network analisys and orgaizational intervention: Insights from a area-wide labor-management committee. Journal of Applied Behavioral Science, v. 22, p. 67-76, 1986. 
A cultura organizacional e a centralidade nas redes sociais: um estudo exploratório em uma empresa de serviços

NELSON, R. E. The strength of strong ties: social networks and intergroup conflict in organizations. The Academy of Management Journal, v. 32, n. 2, p 377401, jun. 1989.

NELSON, R. E. On the shape of verbal networks in organizations. Organization Studies, v. 22, p. 797-824, 2001.

NELSON, R. E.; GOPALAN, S. Do organizational cultures replicate national cultures? Organization Studies, v. 24, p. 115-152, 2003.

NELSON, R. E.; HSU S. Social networks and knowledge management. The encyclopedia of knowledge management, Hershey, p. 826-832, 2005.

NELSON, R. E. Cultura empresarial e atendimento superior - Gerenciando a prestação de services no secúlo 21. Sorocaba: Cidade, 2006.

NELSON, R. E.; VASCONCELOS, E. P. G. O ambiente e o papo: Comparações internacionais e tipologia das redes verbais nas organizações. In: Encontro Anual da ANPAD, XXXI, Rio de Janeiro, Anais... Rio de Janeiro, ANPAD, 2007.

RODRIGUES, S. B. Cultura corporativa e identidade: desinstitucionalização em empresa de telecomunicações brasileira, RAC, São Paulo, v. 1, n. 2, p. 45-72, maio/ago. 1997.

SANTOS, N. M. B. F. dos. Cultura e desempenho organizacional: um estudo empírico em empresas brasileiras do setor têxtil. RAC, São Paulo, v. 2, n. 1, p. 47-66, jan./abr. 1998.

SCHEIN, E. H. Coming to a new awareness of organizational culture. Sloan Management Review, v. 12, p. 3-16, Winter 1984.

SCHEIN, E. H. Organizational Culture and Leadership. San Francisco: JosseyBass, 1992.

SIEGEL, S.; CASTELLAN JR., N. J. Estatística não-paramétrica para ciências do comportamento. 2. ed. Porto
Alegre: Artmed, 2006.

SILVA, L. M. T.; MEDEIROS, C. A. F.; ALBUQUERQUE, L. G. Cultura organizacional e qualidade em serviços. In: SEMEAD - Seminários em Administração FEA/USP, XI, 2008, São Paulo. Anais... São Paulo, FEA-USP, 2008.

STEPHENSON, K; ZELEN, M. Rethinking centrality: methods and examples. Social Networks, v.11, n.1, p.137, Mar. 1989.

TAMAYO, A.; GONDIM, M.G.C. Escala de valores organizacionais. Revista de Administração, v. 31, n. 2, abril/junho 1996.

TÉVENET, M. A Cultura de empresa hoje em dia. Trad. Rosalra Eichenberz. Revista de Administração, São Paulo, v. 26, n. 2, p. 32-39, abril/junho1991.

ZHU. B., WATTS S., CHEN H. Visualizing social network concepts. Decision Support Systems, n. 49, p. 151-161, Fev., 2010. 